\title{
Electrophysiological correlates of reading the single- and interactive-mind
}

\author{
Yi-Wen Wang ${ }^{1}$, Yu-Wei Zheng ${ }^{1}$, Chong-De Lin ${ }^{2 *}$, Jie Wu' and De-Li Shen ${ }^{1 *}$ \\ Academy of Psychology and Behavior, Tianjin Normal University, Tianjin, China \\ 2 State Key Laboratory of Cognitive Neuroscience and Learning, Beijing Normal University, Beijing, China
}

Edited by:

Hans-Jochen Heinze, University of Magdeburg, Germany

Reviewed by:

Fernando MaEstú, Complutense

University, Spain

Lutz Jäncke, University of Zurich,

Switzerland

*Correspondence:

Chong-De Lin and De-Li Shen,

Academy of Psychology and Behavior,

Tianjin Normal University, 241\# Weijin

Road, Tianjin 300074, China.

e-mail: linchongde@263.net;

wangeven@126.com
Understanding minds is the cognitive basis of successful social interaction. In everyday life, human mental activity often happens at the moment of social interaction among two or multiple persons instead of only one-person. Understanding the interactive mind of two- or multi-person is more complex and higher than understanding the single-person mind in the hierarchical structure of theory of mind. Understanding the interactive mind maybe differentiate from understanding the single mind. In order to examine the dissociative electrophysiological correlates of reading the single mind and reading the interactive mind, the 64 channels eventrelated potentials were recorded while 16 normal adults were observing three kinds of Chinese idioms depicted physical scenes, one-person with mental activity, and two- or multi-person with mental interaction. After the equivalent N400, in the 500- to 700-ms epoch, the mean amplitudes of late positive component (LPC) over frontal for reading the single mind and reading the interactive mind were significantly more positive than for physical representation, while there was no difference between the former two. In the 700- to 800-ms epoch, the mean amplitudes of LPC over frontal-central for reading the interactive mind were more positive than for reading the single mind and physical representation, while there was no difference between the latter two. The present study provides electrophysiological signature of the dissociations between reading the single mind and reading the interactive mind.

Keywords: event-related potential, theory of mind, mentalizing, mind-reading, late positive component, Chinese idiom

\section{INTRODUCTION}

Theory of mind (ToM) refers to understanding other's mental states, such as beliefs, intentions, emotions, and thoughts, to predict other's behaviors. ToM is also called mentalizing, or mind-reading, which is a major ingredient in successful social interactions (Frith and Frith, 1999). Wimmer and Perner (1983) investigated the representation of wrong beliefs in young children. Since then, studies concerning ToM have largely been driven by questions arising from the field of cognitive neuroscience. Most of the current studies have been focusing on the understanding of the individual mental state. However, we often need to understand two or multi-person minds during social interaction in real life. Based on the hierarchical hypothesis about ToM, understanding the single mind and understanding the interactive mind might be two different levels of ToM processing.

In the field of cognitive neuroscience, researchers often compared reading the single mind (e.g., desire, belief, and intention, etc,) with the non-mental representation. Belief stories and photograph stories were used in an event-related potential (ERP) study to investigate electrophysiological correlates of reasoning belief and non-mental representations (Sabbagh and Taylor, 2000). Results indicated that ERP elicited by these two tasks differed beginning at $300 \mathrm{~ms}$ post-stimulus: beliefs were associated with an enhanced positivity over left-frontal sites and a stronger negativity over left parietal sites. The "ToM cartoons" (cartoons involving people and also requiring ToM for comprehension), "person cartoons" (cartoons involving people), and "scene cartoons" (cartoons of scenes without people) were also used in a previous ERP study to explore the time course of person perception and ToM (Wang et al., 2010). They found the peak amplitudes of P200 for person perception and ToM cartoons were significantly more positive than for scene cartoons. During 1000-1300 ms epochs, the mean amplitude of late positive component (LPC) for person perception was more positive than for scene representation, while LPC for ToM was more positive than for person perception. But this study contains a number of shortcomings. Firstly, "person cartoons" and "ToM cartoons" consisted of pictures with one or two persons. The processing of different numbers of people could influence the ERP waveforms, which might affect the experimental results. Secondly, the one-person figures and two-person figures were mixed up in those ToM cartoons. As a result, the above study has not revealed the difference between understanding the one-person mind and understanding interactive minds (Wang et al., 2010). The present study differentiated two kinds of ToM: understanding single-person mind and understanding the interactive mind. Our experiment materials were Chinese idioms, which can guarantee the consistency of the material in visual form.

Many electrophysiological studies were carried out to investigate the dynamic processing of understanding the single mind and revealed the time course of the processing (Liu et al., 2004, 2009a,b; Sabbagh et al., 2004; Wang et al., 2008). In an ERP study, participants were asked to perform an "unexpected transform" task with cartoon animations (Liu et al., 2004). A late ERP component (peaking around $800 \mathrm{~ms}$ post-stimulus) with a left-frontal scalp 
distribution, which was inconsistent with a source possibly in the left orbitofrontal cortex, differentiated judgments about belief and reality. The false-belief paradigm includes two classic tasks: unexpected-transfer and deceptive-appearance. The unexpected-transfer paradigm was used to study the time course of ToM (Liu et al., 2004). The deceptive-appearance paradigm was used to explore the brain electrophysiological activity with false-belief reasoning and true-belief reasoning in adults (Wang et al., 2008). Compared with true-belief, false-belief reasoning elicited significant declined late negative component (LNC) in the time window from 400 to $800 \mathrm{~ms}$. Both of the two studies we mentioned above used first-order falsebelief task. However, Liu et al. (2009a) adopted diverse-desires, diverse-beliefs, and diverse-physical judgment tasks to investigate neural system of desire and belief judgment. They found that a mid-frontal late slow wave (LSW) was associated with desire and belief judgments and a right-posterior LSW was only associated with belief judgments. Most of the studies on the research of ToM were done in adults. However, a study has made comparisons of neural correlates of ToM between adults and children (Liu et al., 2009b). They found that in adults, a LSW, with a left-frontal scalp distribution, was associated with reasoning about beliefs. This LSW was also observed in children who could correctly reason about the characters' beliefs but not in children who failed false-belief questions. In almost all of studies on the neural correlates of ToM, the participants were asked to reason about false belief, desire or intention. However, Sabbagh et al.'s (2004) study on the neural systems underlying mental-state decoding showed that decoding mental states from pictures of eyes was associated with an N270-400 component over inferior frontal and anterior temporal regions of the right hemisphere. In summary, we can conclude that the processing of understanding single-person mind was related to the LSW.

The mentioned above studies about the electrophysiological correlates of ToM were mostly to understand a one-person mind, however "understanding of the human mind can be further deepened by moving from one-person neuroscience toward two- and multiperson neuroscience, both conceptually and experimentally." (Hari and Kujala, 2009; p470). We suppose that the ToM consists of a hierarchical framework. Understanding a one-person mind and understanding an interactive mind were different levels of ToM, and the level of understanding an interactive mind is higher than understanding a one-person mind. Consequently, the present study was to compare the differences between understanding the single mind and understanding the interactive mind. Social interaction is a central concept to understanding the nature of social life. Social interaction involves communication in all its forms, such as cooperation, competition, helping, playing, informing, questioning, negotiating, bargaining, voting, and bluffing (Hari and Kujala, 2009). A study indicated that during the "live" interaction, as compared to the recorded condition (watched a video of the interaction), greater activation was seen in brain regions involved in right temporo-parietal junction (TPJ), anterior cingulated cortex and so on (Redcay et al., 2010). Another study sought to characterize neural processes related to aspects of social cognition and empathy. The results found the medial prefrontal cortex (MPFC), temporal lobe, precuneus, occipital lobe showed an increased level of activation for the stimuli with two persons compared to stimuli with only one-person (Krämer et al., 2010). Leng and Zhou
(2010) explored to what extent the brain activity was modulated by the interpersonal relationship between the individual and the other agent, who could be a friend or a stranger. They found the P300 was modulated by the interpersonal relationship between the observer and the other agent. Social cognition suggested that self-projection was the basis of reading the single mind. Perceivers may construct a mental representation of a target's experience, predict the kinds of thoughts and feelings they may have in such a situation and then assume that the target of mentalizing will think or feel much the same thing (Mitchell, 2009). Some scholars believed that understanding of interactive behavior and mind can be explained in the frame of "shared action representation" theory (Sebanz et al., 2006; Knoblich and Sebanz, 2008). This theory indicated that we can only accurately understanding the interactive mind on the basis of representing all individuals' contributions to an action and understanding the relations between persons and their actions in the interaction. According to the hypothesis, we supposed that understanding an interactive mind was more complex than understanding a single mind. And we also wanted to explore the differences of the dynamic processing between understanding an interactive mind and understanding a single mind. The processing of reading interactive mind exists in such two situations. First, the individual, as one of the participants in the interpersonal interaction, responds to other persons' behaviors and minds. Second, the individual infers others' mental states while observing their interactive activities. The present study belongs to the second situation.

Similar to cartoon or picture, the verbal material/story was often used as experimental stimuli in the previous ToM studies (Saxe and Powell, 2006; Abraham et al., 2008, 2010; Liu et al., 2009a). The present study employed the Chinese four-character idioms (cheng-yu) as visual stimuli to convey the descriptive content to ToM. Chinese four-character idioms are brief and concise expressions established by long-term usage and recognized through social practices (Yu et al., 2006). Many idioms describe one-person's mental activity, for example, zi-gao-fen-yong (in Chinese:自告奋勇), which in English means one-person volunteers to do something. Another example depicting the interactive minds of social interaction is fu-chang-fusui (in Chinese: 夫唱妇随) and its English translation is harmony between husband and wife. Consequently, Chinese four-character idioms are suitable verbal materials to study the ToM and we can investigate reading the single mind and reading the interactive mind by reading different types of Chinese four-character idioms. Previous studies mostly have focused on the electrophysiological correlates of reasoning desire, belief, and intention, but the present study has been made on the investigation of the neural dynamics that underlie understanding mental state from Chinese idioms.

Using three types of Chinese four characters idioms - "physical representation idioms," "the single mind idioms," and "the interactive-mind idioms," the present study was designed to explore the dissociative electrophysiological correlates between reading the single mind and reading the interactive mind. Based on previous fMRI results that the MPFC, temporal lobe, precuneus, occipital lobe showed an increased level of activation for the stimuli with two persons compared to stimuli with only one-person (Krämer et al., 2010) and previous ERPs study that the mean amplitude of LPC for ToM was more positive than for person perception (Wang 
et al., 2010), we hypothesized that the amplitude of ERP related to reading the interactive mind would be larger than that of reading the single mind, and that the amplitude of ERP related to reading the single mind would be larger than that of physical representation. According to the study (Wang et al., 2010), they found the P200 for the ToM and person perception conditions was significantly larger than that for the scene condition; then, the LPC amplitude elicited by ToM was larger than that induced by person perception. We proposed that the ERP component at an earlier period would be associated with reading the single mind that separates from that of physical representation, and that the ERP component at later period would be associated with understanding the interactive mind that separates from that of understanding the single mind.

\section{MATERIALS AND METHODS PARTICIPANTS}

Twenty graduate or undergraduate students (mean age $=22$ years; range $=19-26$ years; 10 males and 10 females) participated in the study for pay. Four participants provided excessive electrophysiological artifact during recording and were excluded from the final sample of sixteen participants (mean age $=23$ years; range $=19-26$ years; seven males and nine females) for ERP analyses. All participants were right-handed, had normal or correctedto-normal vision, and reported no history of organic or acquired brain damage. Informed written consent was obtained from all participants prior to EEG recording.

\section{STIMULI AND PROCEDURE}

In the study we grouped Chinese idioms into three types: the first type was physical representation idiom, which described natural scenery or living environment. For example, chong-shan-jun-ling (in Chinese:崇山峻岭), which in English means lofty and precipitous peaks. The second type was reading the single mind idiom, which described a single personal activity and their typical themes were about people's feelings or behavior. For example, gu-ku-lingding (in Chinese:狐苦侉仃), which in English means friendless and wretched. The third type was reading the interactive-mind idiom, which described the interaction of two or more people and their typical themes were about competition, cooperation, help, love, and so on. For example, fu-chang-fu-sui (in Chinese:夫唱妇随).

First, we found all types of idioms in the "Dictionary of Chinese Idioms." Then we arranged all idioms at random. Afterward, we found 14 college students to judge which kind the idioms were. Finally, we selected 70 idioms each kind based on the students' judgment. All the idioms were presented as picture. They were of uniform size $(4.5 \mathrm{~cm}$ in width, $1.2 \mathrm{~cm}$ in height), and each photograph was $960 \times 720$ pixels. Each idiom is Song font and its font size was 26. Idioms in white color were presented in the centerfield of a black background. The participants watched the experimental stimuli subtending a visual angle of approximate $3^{\circ}$. We also matched the word stroke number, the term frequency, the word sentimental color (commendatory term or derogatory term; shown in Table 1): stroke number: $F(2,209)=0.19, p>0.05$; term frequency: $F(2,209)=1.93, p>0.05$; sentimental color: $F(2$, $209)=2.23, p>0.05$. In addition, we also found two types of probe words: a category of words described the form of scene and the other described people's mentality; each category consisted of
Table 1 | Lexical-statistical properties for three types of Chinese idioms.

\begin{tabular}{llll}
\hline Idioms' types & $\begin{array}{l}\text { Stroke } \\
(\mathbf{M} \pm \mathbf{S D})\end{array}$ & Frequency & $\begin{array}{l}\text { Sentimental } \\
\text { color (M } \pm \mathbf{S D} \text { ) }\end{array}$ \\
\hline $\begin{array}{l}\text { Physical } \\
\text { representation }\end{array}$ & $30.39 \pm 6.58$ & 48 & $2.27 \pm 0.72$ \\
$\begin{array}{l}\text { Reading the } \\
\text { single mind }\end{array}$ & $30.10 \pm 7.81$ & 83 & $2.03 \pm 0.82$ \\
$\begin{array}{l}\text { Reading the } \\
\text { interactive mind }\end{array}$ & $29.67 \pm 6.29$ & 96 & $2.04 \pm 0.75$ \\
\hline
\end{tabular}

Chinese idiom frequency is measured as a word-per-million figure using the People's daily Character Frequency Statistics (1998).

17 words. All words were nouns. The scene probe words included narrowness, expansion, majesty, and so on, and the mental probe words included pleasure, relaxation, sorrow, and so on.

The time course of a trial was as follows: First, the fixation point appeared in the center of the screen for $500 \mathrm{~ms}$ indicating the start of a new trial. Then, participants saw a screen with an idiom for $1500 \mathrm{~ms}$. Finally, a probe word was present for $2000 \mathrm{~ms}$. The ITI among the trials were randomized from 400 to $600 \mathrm{~ms}$. In the experiment, participants were required to understand the meaning of the Chinese idioms, and imagine the scene shape or reason the people's mentality in idioms. Then, they judged whether the contents of the Chinese idioms were consistent with the contents of the probe words by pressing a number key. The inconsistent probe words were opposite to the contents of the idioms, which ensured the participants to understand the meaning of the idioms when they pressed the inconsistent key. The experiment consisted of 210 trials and each of three categories of the idioms included 70 trials. The experiments were divided into two blocks, with 105 trials of approximately $8 \mathrm{~min}$ in each block. The idioms were presented in pseudo-random order. The experimental order was balanced among participants. The responses to three types of cartoons were counterbalanced between participants to avoid the sequence effects. Before the formal experiment, participants were first to have practice experiments that consist of 15 trials. These trials were similar but different from the following formal session in the ERP experiment.

\section{ELECTROPHYSIOLOGICAL RECORDING AND ANALYSIS}

The electroencephalogram (EEG) was recorded from 64 scalp sites using $\mathrm{Ag}-\mathrm{AgCl}$ electrodes mounted in an elastic cap according to the International 10/20 System. All electrode recordings were referenced to an electrode placed at the right mastoid, and the EEG data were re-referenced off-line to linked mastoid electrodes by subtracting from each sample of data recorded at each channel one-half the activity recorded at the left mastoid. The horizontal electro-oculogram (EOG) was recorded from electrodes placed $1.5 \mathrm{~cm}$ lateral to the left and right external canthi. Eye blinks were recorded from left supraorbital and infraorbital electrodes. All interelectrode impedance was maintained below $5 \mathrm{k} \Omega$. EEG and EOG were amplified using a 0.05 to $100-\mathrm{Hz}$ band pass and continuously sampled at $1000 \mathrm{~Hz} / \mathrm{channel}$ for off-line analysis. EOG artifacts of eyes blink and horizontal move were revised from all trials automatically using Scan software. EEG contaminated with artifacts due to amplifier clipping, bursts of electromyographic (EMG) activity, 
or peak-to-peak deflection exceeding $\pm 75 \mu \mathrm{V}$ were excluded from trials. In the present study, EEG of idiom representation period was selected for critical analysis, and only the trials that subjects correctly responded were overlapped and averaged. The ERP were time-locked to the onset of idiom representation. The data were transformed off-line to epochs of -200-1000 ms. According to the previous studies (Sabbagh and Taylor, 2000; Liu et al., 2009a) and the ERP data of present study, the nine electrode sites were chosen for statistical analysis. The electrodes in a $3 \times 3$ grid encompassing scalp locations from left to right (laterality) and from anterior to posterior (caudality) were: F3, Fz, F4, C3, Cz, C4, P1, Pz, and P2. The amplitudes of P200 and N400 were measured from baseline to peak, and the mean amplitudes of the LPC were measured in $100 \mathrm{~ms}$ intervals over 500-1000 ms. The ERP components were analyzed using three-way repeated-measures analysis of variance (ANOVAs): condition (physical representation, reading the single mind, reading the interactive mind), laterality (left, midline, right), caudality (frontal, central, parietal). When necessary, for all of our analyses, $p$ values were adjusted using the Greenhouse-Geisser correction. Bonferroni correction was used for multiple comparisons.

\section{RESULTS}

\section{BEHAVIORAL PERFORMANCE}

There were no significant differences in the participants' accuracy for physical representation, reading the single mind, and reading the interactive-mind Chinese idioms (92\%, 91\%, and 91\% correct, respectively), $F(2,45)=0.43, p=0.65$. This indicated that the difficulty of three conditions was equivalent. There were no significant differences in the participants' reaction time for three conditions either $(850.61 \pm 187.61 \mathrm{~ms}, 885.87 \pm 212.33 \mathrm{~ms}, 846.76 \pm 197.90 \mathrm{~ms}$ reaction time, respectively), $F(2,45)=0.19, p=0.83$.

\section{ERP RESULTS}

We designed the physical representation condition to provide a control baseline. Therefore, any differences in waveforms between the reading the single mind and physical representation conditions reveals the components associated with reading the single mind, and any differences in waveforms between the reading the interactive-mind and physical representation conditions revealed the components associated with reading the interactive mind. Any difference in waveforms between the reading the single mind and reading the interactive-mind conditions revealed the components that differed between reading the single mind and reading the interactive mind. Figure 1 displays the grand average waveforms for all three conditions from nine electrodes: $\mathrm{F} 3, \mathrm{Fz}, \mathrm{F} 4, \mathrm{C} 3, \mathrm{Cz}$, $\mathrm{C} 4, \mathrm{P} 1, \mathrm{Pz}$, and $\mathrm{P} 2$. After artifact rejection, the mean numbers of valid trials were 54, 54, and 53 epochs used in the ERP averaging for physical representation, reading the single mind, and reading the interactive-mind condition.

It was clear from visual inspections of the waveforms in Figure 1 that there was a LPC differentiation between the reading the single mind (more positive) and physical representation conditions and that there was also a LPC differentiation between the reading the interactive-mind (more positive) and physical representation conditions. These differentiations were around $500 \mathrm{~ms}$ post-stimulus. To ensure this, mean amplitude in the 500 - to 700 -ms post-stimulus epoch was computed for each condition from all electrodes in the $3 \times 3$ grid of scalp locations. Visual inspections of the waveforms in Figure 1 also suggested that there was an effect of condition around 700-900 ms post-stimulus. To ensure this, mean amplitudes in the 700 - to 800 and 800 - to 900 -ms post-stimulus epochs were computed for each condition from all electrodes in the $3 \times 3$ grid of scalp locations. The results indicated that there were no

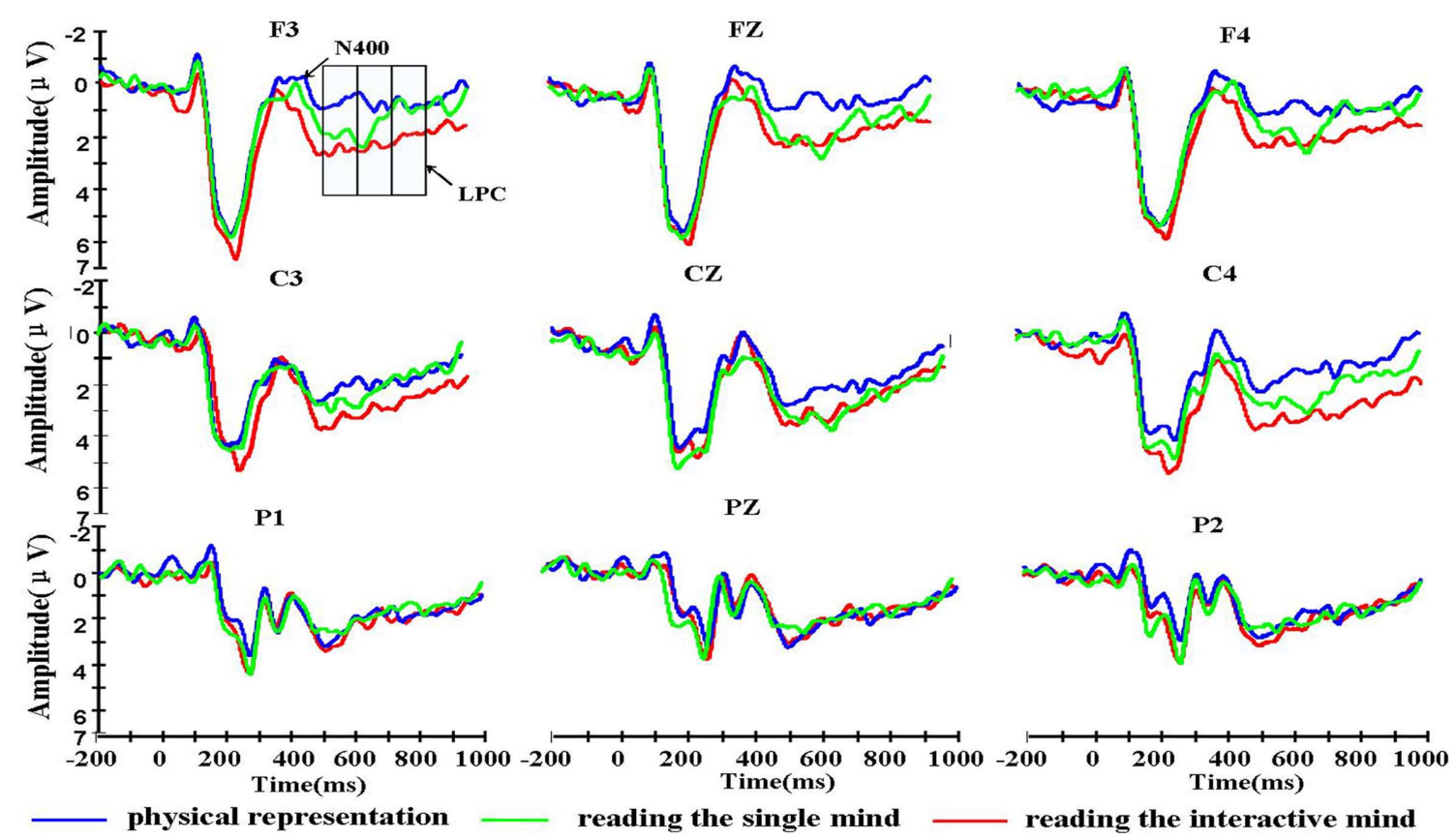

FIGURE 1 | Grand average event-related brain potential waveforms for the three types of Chinese idioms of physical representation, reading the single mind, and reading the interactive mind from nine electrodes. 
differences among three task conditions in P200 $[F(2,30)=1.95$, $\mathrm{MSE}=10.55, p=0.16]$, and $\mathrm{N} 400[F(2,30)=1.23, \mathrm{MSE}=7.15$, $p=0.31$; shown in Table 2].

\section{Late positive component}

A 3 (condition) $\times 3$ (laterality) $\times 3$ (caudality) repeated-measures ANOVA was conducted on the mean amplitudes of single electrodes in the $3 \times 3$ grid of scalp locations for the 500 - to 600 -ms poststimulus epochs. The results of the ANOVAs showed that there was not a significant two-way interaction between condition and laterality, $F(4,60)=0.69$, MSE $=0.60, p=0.557$, nor a significant three-way interaction between condition, laterality, and caudality, $F(8,120)=0.49, \mathrm{MSE}=0.58, p=0.777$. There were moderately significant main effects of task condition, $F(2,30)=3.19$, MSE $=10.45$, $p=0.056$. We also found there was a significant two-way interaction between condition and caudality, $F(4,60)=7.86, \mathrm{MSE}=1.72$, $p=0.001$ (shown in Figure 2). Follow-up means contrasts revealed that in the frontal area, there were significant differences between the conditions, $F(2,30)=8.90, p=0.001$. Bonferroni-corrected pairwise comparisons showed that there were significant differences between the reading the single mind $(1.71 \pm 0.77 \mu \mathrm{V})$ and physical representation condition $(0.60 \pm 0.64 \mu \mathrm{V}), p=0.04$, and there were significant differences between the reading the interactive-mind $(2.17 \pm 0.75 \mu \mathrm{V})$ and physical representation condition $(0.60 \pm 0.64 \mu \mathrm{V}), p=0.002$, but there was no significant difference between reading the single mind $(1.71 \pm 0.77 \mu \mathrm{V})$ and reading the interactive mind $(2.17 \pm 0.75 \mu \mathrm{V}), p=0.736$. We also found in the central area where there were significant differences between the conditions, $F(2,30)=4.13, p=0.026$. Bonferroni-corrected pairwise comparisons showed that there were significant differences between the reading the interactive-mind $(3.22 \pm 0.67 \mu \mathrm{V})$ and physical representation condition $(2.07 \pm 0.67 \mu \mathrm{V}), p=0.042$, but there was no significant differences between the reading the single mind $(2.67 \pm 0.79 \mu \mathrm{V})$ and physical representation condition

Table 2 | ANOVA of P200, N400, and the LPC amplitudes measured at $100 \mathrm{~ms}$ intervals during the 500- to 1000-ms period.

\begin{tabular}{|c|c|c|c|c|c|c|}
\hline \multirow{2}{*}{$\begin{array}{l}\text { Time window } \\
\text { (ms) }\end{array}$} & \multirow{2}{*}{$\begin{array}{l}\text { Condition main } \\
\text { effects } F(2,30)\end{array}$} & \multirow{2}{*}{$\begin{array}{l}\text { Condition } \times \text { Caudality } \\
\text { interaction effects } F(4,60)\end{array}$} & \multicolumn{3}{|c|}{$\mathrm{M} \pm \mathrm{SE}$ (amplitudes, $\mu \mathrm{V}$ ) } & \multirow[t]{2}{*}{ Post hoc test } \\
\hline & & & $\begin{array}{l}\text { Physical } \\
\text { representation }\end{array}$ & $\begin{array}{l}\text { Reading the } \\
\text { single mind }\end{array}$ & $\begin{array}{l}\text { Reading the } \\
\text { interactive mind }\end{array}$ & \\
\hline $\mathrm{P} 200$ & 1.954 & 1.372 & $5.61 \pm 0.47$ & $6.21 \pm 0.57$ & $6.30 \pm 0.49$ & $1-2,2-3,1-3$ \\
\hline N400 & 1.232 & 1.455 & $-1.59 \pm 0.58$ & $-1.29 \pm 0.54$ & $-1.14 \pm 0.51$ & $1-2,2-3,1-3$ \\
\hline $600-700$ & $5.035^{*}$ & $6.546^{* *}$ & $1.58 \pm 0.62$ & $2.74 \pm 0.71$ & $2.96 \pm 0.59$ & $1-2,2-3,1-3^{*}$ \\
\hline $700-800$ & 2.847 & $13.45^{*}$ & $1.44 \pm 0.56$ & $1.90 \pm 0.64$ & $2.67 \pm 0.56$ & $1-2,2-3,1-3^{*}$ \\
\hline $800-900$ & 1.13 & 2.122 & $1.14 \pm 0.42$ & $1.31 \pm 0.56$ & $1.71 \pm 0.44$ & $1-2,2-3,1-3$ \\
\hline $900-1000$ & 0.955 & 1.556 & $0.87 \pm 0.40$ & $1.00 \pm 0.53$ & $1.39 \pm 0.40$ & $1-2,2-3,1-3$ \\
\hline
\end{tabular}

In the post hoc test, the number 1 physical representation, 2 reading the single mind, and 3 reading the interactive mind. $\left({ }^{*}{ }^{*} P<0.001,{ }^{*} P<0.01,{ }^{*} P<0.05\right)$.
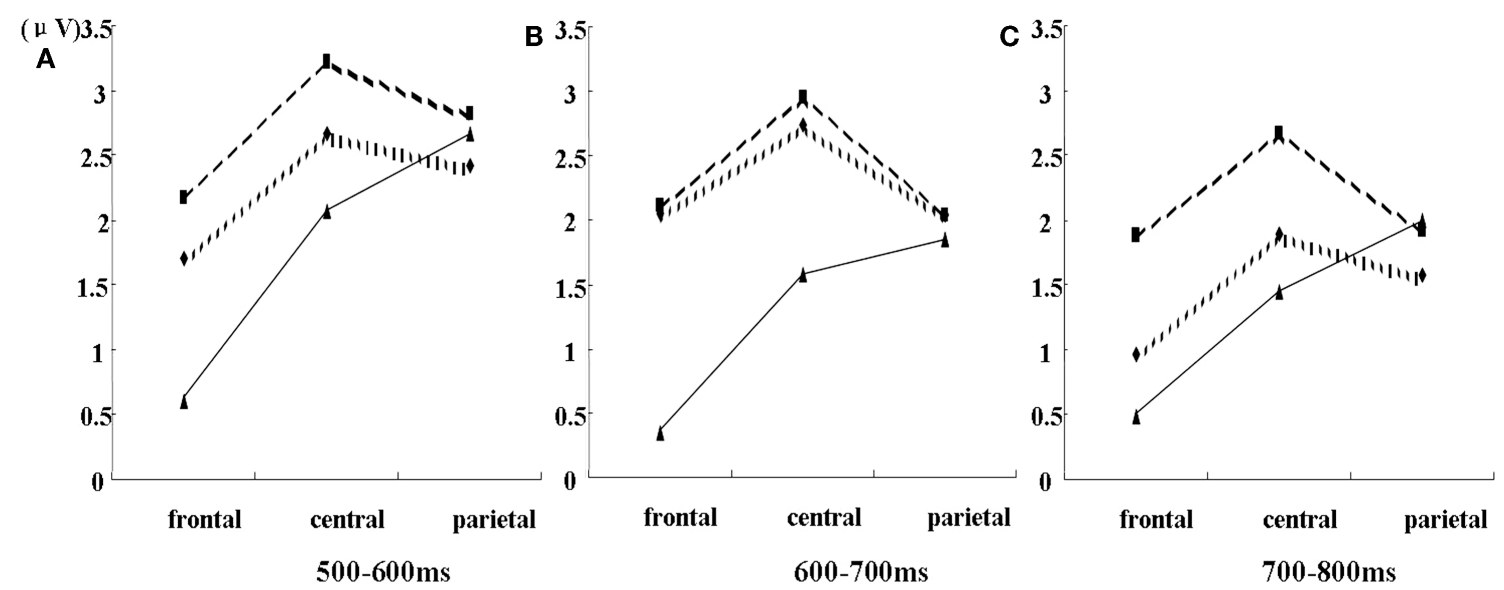

reading the single mind

reading the interactive mind

physical represent ation

FIGURE 2 | (A) Interaction between condition and caudality in 500-600 ms. (B) Interaction between condition and caudality in 600-700 ms. (C) Interaction between condition and caudality in 700-800 ms. 
$(2.07 \pm 0.67 \mu \mathrm{V}), p=0.302$, and there was no significant difference between reading the single mind $(2.67 \pm 0.79 \mu \mathrm{V})$ and reading the interactive mind $(3.22 \pm 0.67 \mu \mathrm{V}), p=0.691$ (shown in Table 2). This result confirms what is observed in the waveforms in Figure 1, a LPC differentiation between the reading the single mind (more positive) and physical representation conditions and a LPC differentiation between the reading the interactive mind (more positive) and physical representation conditions. This pattern of results suggests that in the 500- to 600-ms epoch, there was a frontal ERP component associated with both reading the single mind and reading the interactive mind. This was further illustrated in Figure 3, which displays the mean amplitude difference between the conditions (physical representation subtracted from reading the single mind) at the $\mathrm{Fz}$ site, as well as the corresponding scalp topographic map. Darker red indicates positive wave of conditions in scalp topographic map.

A 3 (condition) $\times 3$ (laterality) $\times 3$ (caudality) repeated-measures ANOVA was conducted on the mean amplitudes of single electrodes in the $3 \times 3$ grid of scalp locations for the 600 - to 700 -ms poststimulus epochs. The results of the ANOVAs showed that there were main effects of task condition, $F(2,30)=5.04, \mathrm{MSE}=11.60$, $p=0.013$. We also found there was a significant two-way interaction between condition and caudality, $F(4,60)=6.55$, MSE $=2.85$, $p=0.003$ (shown in Figure 2). Follow-up means contrasts revealed that in the frontal area, there were significant differences between the conditions, $F(2,30)=11.53, p<0.001$. Bonferroni-corrected pairwise comparisons showed that there were significant differences between the reading the single mind $(2.05 \pm 0.70 \mu \mathrm{V})$ and physical representation condition $(0.35 \pm 0.57 \mu \mathrm{V}), p=0.014$, and there were significant differences between the reading the interactive mind $(2.11 \pm 0.63 \mu \mathrm{V})$ and physical representation condition $(0.35 \pm 0.57 \mu \mathrm{V}), p<0.001$, but there was no significant difference between reading the single mind $(2.05 \pm 0.70 \mu \mathrm{V})$ and reading the interactive mind $(2.11 \pm 0.63 \mu \mathrm{V}), p=1.000$. We also found in

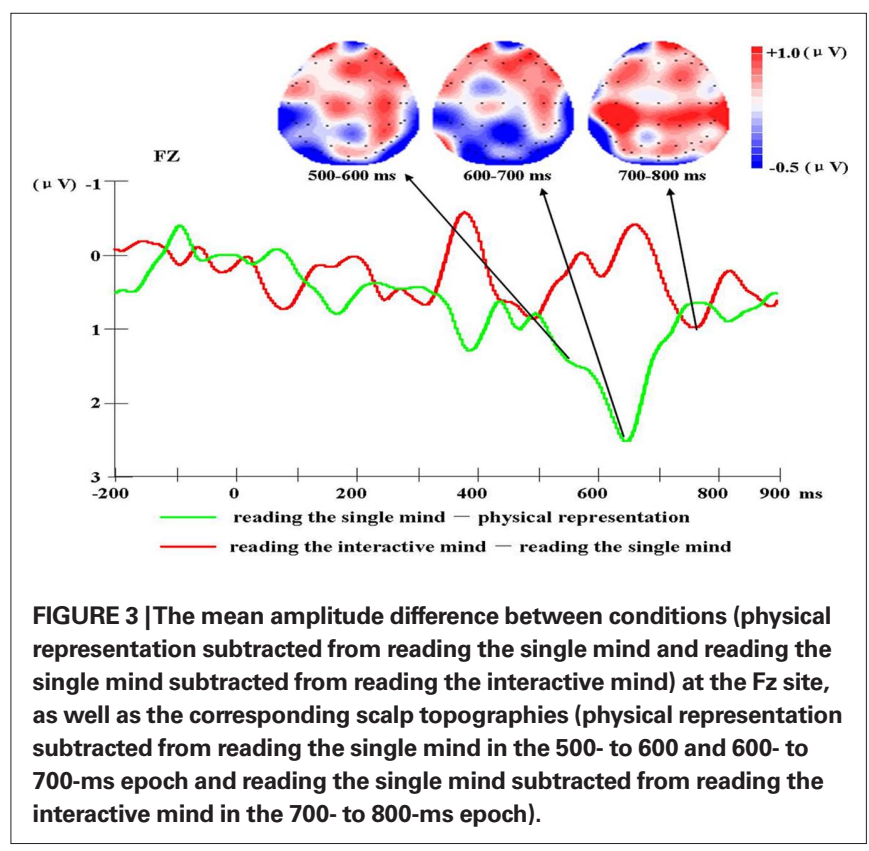

the central area where there were significant differences between the conditions, $F(2,30)=6.09, p=0.006$. Bonferroni-corrected pairwise comparisons showed that there were significant differences between the reading the single mind $(2.74 \pm 0.71 \mu \mathrm{V})$ and physical representation condition $(1.58 \pm 0.62 \mu \mathrm{V}), p=0.044$, and there were significant differences between the reading the interactive mind $(2.96 \pm 0.59 \mu \mathrm{V})$ and physical representation condition $(1.58 \pm 0.62 \mu \mathrm{V}), p=0.030$, but there was no significant difference between reading the single mind $(2.74 \pm 0.71 \mu \mathrm{V})$ and reading the interactive mind $(2.96 \pm 0.59 \mu \mathrm{V}), p=1.000$ (shown in Table 2). This result confirms what is observed in the waveforms in Figure 1, a LPC differentiation between the reading the single mind (more positive) and physical representation conditions and a LPC differentiation between the reading the interactive mind (more positive) and physical representation conditions. The results of the ANOVAs showed that there was not a significant two-way interaction between condition and laterality, $F(4,60)=1.36, \mathrm{MSE}=0.60, p=0.267$, nor a significant three-way interaction between condition, laterality, and caudality, $F(8,120)=0.42, \mathrm{MSE}=0.71, p=0.829$. This pattern of results suggested that in the 600- to 700 -ms epoch, there was a frontal-central ERP component associated with both reading the single mind and reading the interactive mind. This was further illustrated in Figure 3, which displays the mean amplitude difference between the conditions (physical representation subtracted from reading the single mind) at the $\mathrm{Fz}$ site, as well as the corresponding scalp topographic map. Darker red indicates positive wave of conditions in scalp topographic map.

A 3 (condition) $\times 3$ (laterality) $\times 3$ (caudality) repeated-measures ANOVA was conducted on the mean amplitudes of single electrodes in the $3 \times 3$ grid of scalp locations for the 700 - to 800 -ms poststimulus epochs. The results of the ANOVAs showed that there were no main effects of task condition, $F(2,30)=2.85$, MSE $=13.66$, $p=0.092$, and there was not a significant two-way interaction between condition and laterality, $F(4,60)=0.68$, MSE $=0.96$, $p=0.57$, nor a significant three-way interaction between condition, laterality, and caudality, $F(8,120)=0.79$, MSE $=1.18, p=0.545$. There was a significant two-way interaction between condition and caudality, $F(4,60)=3.85$, MSE $=3.50, p=0.024$ (shown in Figure 2). Follow-up means contrasts revealed that in the frontal area, there were significant differences between the conditions, $F(2,30)=5.54, p=0.009$. Bonferroni-corrected pairwise comparisons showed that there were significant differences between the reading the interactive mind $(1.88 \pm 0.56 \mu \mathrm{V})$ and reading the single mind condition $(0.96 \pm 0.56 \mu \mathrm{V}), p=0.019$, and there were significant differences between the reading the interactive mind $(1.88 \pm 0.56 \mu \mathrm{V})$ and physical representation condition $(0.48 \pm 0.46 \mu \mathrm{V}), p=0.017$, but there was no significant difference between reading the single mind $(0.96 \pm 0.56 \mu \mathrm{V})$ and physical representation $(0.48 \pm 0.46 \mu \mathrm{V}), p=1.000$. We also found in the central area where there were significant differences between the conditions, $F(2,30)=5.02, p=0.013$. Bonferroni-corrected pairwise comparisons showed that there were significant differences between the reading the interactive-mind $(2.67 \pm 0.56 \mu \mathrm{V})$ and physical representation condition $(1.44 \pm 0.56 \mu \mathrm{V}), p=0.027$, and there were moderately significant differences between the reading the interactive-mind $(2.67 \pm 0.56 \mu \mathrm{V})$ and reading the single mind condition $(1.90 \pm 0.64 \mu \mathrm{V}), p=0.085$, but there was no significant 
differences between the reading the single mind $(1.90 \pm 0.64 \mu \mathrm{V})$ and physical representation conditions $(1.44 \pm 0.56 \mu \mathrm{V}), p=0.950$ (shown in Table 2). This result confirms what is observed in the waveforms in Figure 1, a LPC differentiation between the reading the interactive mind (more positive) and physical representation conditions and a LPC differentiation between the reading the interactive mind (more positive) and reading the single mind conditions. This pattern of results suggested that in the 700- to 800-ms post-stimulus epoch, there was a frontal-central ERP component associated with reading the interactive mind only but not reading the single mind. This was further illustrated in Figure 3, which displays the mean amplitude difference between the conditions (reading the single mind subtracted from reading the interactive mind) at the Fz site, as well as the corresponding scalp topographic map. Darker red indicates positive wave of conditions in scalp topographic map. In the 800 - to $900-\mathrm{ms}$, there were no main or interaction effect of any of the condition contrasts. Finally, there were no significant differences between the conditions at any locations for any of the epochs. Thus, the effects of conditions appeared in the 500- to 600,600 - to 700 , and 700 - to 800 -ms.

\section{DISCUSSION}

\section{THEORY OF MIND VERSUS PHYSICAL REPRESENTATION}

The present study was designed to explore the time course of the dissociation between reading the single mind and reading the interactive mind. The ERP results revealed that in 500-700 ms, the mean amplitudes of LPC over frontal for reading the single mind and reading the interactive mind was significantly greater than that for physical representation, while there was no difference between the former two. The above results suggest that individuals can distinguish mental processing from non-mental processing in 500-700 ms. Sabbagh and Taylor (2000) indicated that ERPs elicited by mental representation and non-mental representation differed in 600-840 ms. Our study also found similar results. These results suggest that individuals can distinguish mental processing from non-mental processing at later post-stimulus stage.

ToM processing depends upon at least two kinds of representation: representation of another person and a representation of that other person's mental state. While representation of a person is a likely prerequisite for ToM, achieving a representation of another's mental state is the core feature of ToM (Leslie, 1999). Reading the single mind and reading the interactive mind both need the representation of person. In this study, the N400 indicated possibly the individual represented person or natural objects and these two processing both belong to the level of consciousness, therefore there were no significant difference among three task conditions in N400. In 500-700 ms, reading the single and interactive mind both need the representation person perception and person's mental states, while physical representation only needs representation natural objects, therefore the mean amplitudes of LPC for reading the single mind and reading the interactive mind were significantly more positive than that for physical representation.

Over the past decade, a highly consistent observation in cognitive neuroscience had been the demonstration that ToM engaged a set of brain regions including MPFC, TPJ, and superior temporal sulcus (STS) as well as temporal poles and amygdale (Frith and Frith, 2001, 2003, 2006; Calarge et al., 2003; Saxe and Kanwisher,
2003; Blakemore et al., 2004; Gallagher and Frith, 2004; Saxe and Wexler, 2005; Amodio and Frith, 2006; Kobayashi et al., 2006; Mitchell, 2006, 2008; Perner et al., 2006; Saxe and Powell, 2006; Sommer et al., 2007; Abraham et al., 2008, 2010; Coricelli and Nagel, 2009). While the previous fMRI studies have been limited by the fact that the temporal resolution of $\mathrm{AMRI}$ is relatively low, the advantage made in the present study is that, by taking advantage of the greater temporal resolution of ERPs, we have revealed the time course by which the physical representation precedes the representation of ToM. Esslen et al. (2008) found in the reflective self condition, only dorsal parts of the MPFC were activated, while the pre-reflective self condition showed strong involvement of the ventral MPFC. Therefore, MPFC plays a predominant role was in the understanding self and understanding other's mind. That is, the MPFC was a necessary component of reasoning of ToM.

A study found the questions of false-belief and true-belief elicited LNC at centro-frontal sites in the 400- to 800-ms epoch (Wang et al., 2008). Another neuroscience study identified a mid-frontal LSW was associated with desire and belief judgments in the 800to 850-ms epoch (Liu et al., 2009a). Wang et al. (2010) indicated a LPC associated with ToM and maximal amplitudes of the LPC at the frontal sites. We also found reading the single mind and reading the interactive-mind conditions elicited more intense and extensive electrical activity than physical representation conditions in the frontal area of the scalp, which is consistent with previous findings. These results demonstrated that a late ERP component over frontal regions was associated with ToM processing. The ToM processing requires several cognitive processes. Firstly, we must perceive the characters. Secondly, based on those perceptions about the person, we can read the others' mind. It is possible that the late ERP component over frontal regions reflect the processes associated with integrating mental representation versus person representation within a given context.

The present study has both similarities with and differences to prior ERP study. In the study Liu et al. (2009b) and Wang et al. (2010), the dissociation of mental representation and non-mental representation emerged at 775-850 ms and 300-1500 ms, whereas in the present study, the dissociation of mental representation and non-mental representation emerged at 500-700 ms. This difference may be due to experimental material. Neuroscience studies of ToM used many experimental paradigms, including the recognition of mental-state terms (Baron-Cohen et al., 1994), stories (Saxe and Powell, 2006; Liu et al., 2009a), single-frame cartoons (Gallagher et al., 2000), comic strip cartoons (Kobayashi et al., 2007a,b; Sommer et al., 2007), and interactive games (Gallagher et al., 2002; Polezzi et al., 2008; Kamarajan et al., 2009; Boksem and De Crener, 2010). The present study task is more difficult than single-frame cartoon task and less difficult than comic strip cartoons task and interactive games task, due to different task difficulty, the results of the present study differed from the previous ERP studies.

\section{READING THE SINGLE MIND VERSUS READING THE INTERACTIVE MIND}

A major finding of the present study is the dissociation between understanding the single mind and understanding the interactive mind. In the 700- to 800-ms epoch, the mean amplitudes of LPC over frontal-central for reading the interactive mind was more positive than that for reading the single mind and physical 
representation, while there was no difference between the latter two. That is, the dissociation between understanding the interactive mind and understanding the single mind occurred in the 700- to 800-ms. As indicated above, we also found a frontal ERP component was associated with both reading the single mind and reading the interactive mind in the 500- to $700-\mathrm{ms}$ epoch. It means that the individual firstly distinguished mental processing from non-mental processing, and then distinguished understanding the single mind from understanding the interactive mind.

Tager-Flusberg and Sullivan (2000) argued that there were two distinct components of ToM: a social-cognitive and a socialperceptual component. The social-cognitive component entails the conceptual understanding of the mind as a representational system, the social-perceptual component includes the capacity to distinguish between people and objects, and to make on-line rapid judgments about people's mental state from their facial and body expressions. Reading the single mind and reading the interactive mind both belong to social-cognitive component and they need to reason about mental state. Reading the interactive mind first needs to reading individual's mind in the interaction, which consists with processing of reading the single mind. In 500-700 ms, reading the interactive mind possibly indicated reading individual's mind in the interaction, therefore there was no significant difference between reading the single mind and reading the interactive mind in 500-700 ms. Sebanz indicated that we can only accurately understanding the interactive mind on the basis of representing all individuals' contributions to an action and understanding the relations between persons and their actions in the interaction, whereas reading the single mind only understands one-person contributes to action. In 700-800 ms, reading the interactive mind possibly processed all individuals' contributions to an action and understood the relations of "the individual and the individual," "the behavior and the behavior" in the interaction. Reading the single mind only understands one-person contributes to action, therefore there were significant difference between reading the interactive mind and reading the single mind in 700-800 ms.

Arguments in favor of brain modules often begin with the assumption that social interaction gives rise to benefits that are associated with the selection pressure necessary for developing specialized brain modules responsible for carrying out domainspecific social-cognitive processes (Adolphs et al., 2001). To some extent, the differences of the LPC between reading the single mind and reading the interactive mind are consistent with this view. It is likely that in $700-800 \mathrm{~ms}$, a cognitive process specialized for reading the interactive mind. Hari and Kujala (2009) emphasized the organism-environment system was important for communications. Understanding the organism-environment system for reading the interactive mind is more complex than for reading the single mind. Therefore, in 700-800 ms, reading the interactive mind recruits higher activity than reading the single mind.

Up to now, researches about the neural basis of social interaction were still in its infancy. Krämer et al. (2010) found that the MPFC, temporal lobe, precuneus, occipital lobe showed an increased level of activation for the stimuli with two persons compared to stimuli with only one-person. To some extent, the present study results are consistent with fMRI findings. These results indicated that reading the single mind and reading the interactive mind are two different processing. Previous neuroimaging researches found several brain areas associated with social interaction. These brain areas includes: MPFC (Iacoboni et al., 2004), precuneus (Iacoboni et al., 2004), paracingulate cortex (Rilling et al., 2004; Walter et al., 2004), amygdale (Spezi et al., 2007). It was clear from Figure 1 that the differentiation between reading the interactive mind and reading the single mind in lateral frontal sites was bigger than in medial frontal sites. A previous study also demonstrated a role for lateral frontopolar cortex in the social domain (Raposo et al., 2010). They found that medial frontopolar cortex was modulated by other judgment relative to self judgment. Lateral frontopolar cortex was significantly activated during relational condition compared to self judgment. The relational condition was a more sophisticated mentalizing task that required participants to compare their own judgments with their beliefs about another person's judgments. The combined results demonstrate that there are several "core" regions - including MPFC, precuneus, paracingulate cortex, amygdale - may contribute to interactive-mind reasoning. Understanding interactive mind needs the interaction of these core regions. Compared to other regions, the MPFC maybe plays a more important role in understanding interactive mind. The likelihood that the MPFC involved in understanding interactive mind is higher than other regions.

We know little about the dynamics processing of social interaction. Almost all of studies about the neuroimaging of social interaction utilized Ultimatum game or monetary gain and loss game (Kamarajan et al., 2009; Mobbs et al., 2009; Boksem and De Crener, 2010; Maarten et al., 2010). And participants were asked to actually participate in the game. These paradigms used money as a feedback stimulus to study people's evaluation on the feedback results and found feedback related negativity (FRN) was associated with monetary gain and loss feedback. However, the present study required participants to understand the interactive mind from Chinese idioms. This may be the reason why the results we have gotten were not consistent with previous researches. We used verbal form-idiom-as experimental material and found that a frontal-central LPC was observed for reading the interactive mind only in 700-800 ms. In summary, we believe that the dynamic processing of reading the interactive mind occurs mainly in the 500 - to 800 -ms, especially in the 700 - to 800 -ms. The present study indicated reading the single mind can be seen to underlie reading the interactive mind. This hierarchical framework support social cognition and interpersonal interaction.

Our data provide a direct comparison between the electrophysiological correlates for reading the single mind as well as reading the interactive mind. Our findings show that reading the interactive mind overlaps the neural system capable of reading the single mind but requires the involvement of an additional system. This suggests a developmental account where people's reading the interactive mind builds upon their reading the single mind. That is, people firstly are able to read the single mind, and the reading the interactive mind builds on that earlier understanding by involving the same mental-state processing characteristic of reading the single mind plus an additional the interactivemind processing as well. We believe that reading the single mind and reading the interactive mind are two different levels of ToM. 
Reading the single mind is the basis for reading the interactive mind and the level of reading the interactive mind is higher than the level of reading the single mind. In the future, understanding social interaction can be further deepened in neuroscience, and our findings will illuminate electrophysiological correlates of social interaction.

\section{REFERENCES}

Abraham, A., Rakoczy, H., Werning, M., Cramon, D. Y., and Schubotz, R. I. (2010). Matching mind to world and vice versa: functional dissociations between belief and desire mental state processing. Soc. Neurosci. 5, $1-18$.

Abraham, A., Werning, M., Rakoczy, H, Cramon, D. Y., and Schubotz, R. I. (2008). Minds, persons, and space: an $\mathrm{AMRI}$ investigation into the relational complexity of higher-order intentionality. Conscious. Cogn. 17, 438-450.

Adolphs, R., Tranel, D., and Damasio, H. (2001). Emotion recognition from faces and prosody following temporal lobectomy. Neuropsychology 15, 396-404.

Amodio, D. M., and Frith, C. D. (2006). Meeting of minds: the medial frontal cortex and social cognition. Nat. Rev. Neurosci. 7, 268-277.

Baron-Cohen, S., Ring, H. A., Moriarty, J., Schmitz, B., Costa, D., and Ell, P. (1994). Recognition of mental state terms: clinical findings in children with autism and a functional neuroimaging study of normal adults. Br. J. Psychiatry 165, 640-649.

Blakemore, S. J., Winston, J., and Frith, U. (2004). Social cognitive neuroscience: where are we heading? Trends Cogn. Sci. 8, 216-222.

Boksem, M. A. S., and De Crener, D. (2010). Fairness concerns predict medial frontal negativity amplitude in ultimatum bargaining. Soc. Neurosci. 5, 118-128.

Calarge, C., Andreasen, N. C., and O'Leary, D.S. (2003).Visualizing how one brain understands another: a PET study of theory of mind. Am. J. Psychiatry 160, 1954-1964.

Coricelli, G., and Nagel, R. (2009). Neural correlates of depth of strategic reasoning in medial prefrontal cortex. Proc. Natl. Acad. Sci. U.S.A. 106,9163-9168.

Esslen, M., Metzler, S., Pascual-Marqui, R., and Jancke, L. (2008). Pre-reflective and reflective self-reference: a spatiotemporal EEG analysis. Neuroimage 42, 437-449.

Frith, C. D., and Frith, U. (1999). Interacting minds - a biological basis. Science 286, 1692-1695.

Frith, C. D., and Frith, U. (2006). The neural basis of mentalizing. Neuron 50, 531-534.
Frith, U., and Frith, C. D. (2001). The biological basis of social interaction. Curr. Dir. Psychol. Sci. 10, 151-155.

Frith, U., and Frith, C. D. (2003). Development and neurophysiology of mentalizing. Philos. Trans. R. Soc. Lond. B Biol. Sci. 358, 459-473.

Gallagher, H. L., and Frith, C. D. (2004). Dissociable neural pathways for the perception and recognition of expressive and instrumental gestures. Neuropsychologia 42, 1725-1736.

Gallagher, H. L., Happe, F., Brunswick, N., Fletcher, P.C., Frith, U., and Frith, C.D. (2000). Reading the mind in cartoons and stories: an fMRI study of "theory of the mind" in verbal and nonverbal tasks. Neuropsychologia 38, 11-21.

Gallagher, H. L., Jack, A. I., Roepstorff, A., and Frith, C. D. (2002). Imaging the intentional stance in a competitive game. Neuroimage 16, 814-821.

Hari, R., and Kujala, M. V. (2009). Brain basis of human social interaction: Physiol. Rev. 89, 453-479.

Iacoboni, M., Lieberman, M. D., Knowlton, B. J., Molnar-Szakacs, I., Moritz, M., Throop, C. J., and Fiske, A. P. (2004). Watching social interactions produces dorsomedial prefrontal and medial parietal BOLD fMRI signal increases compared to a resting baseline. Neuroimage 21 , 1167-1173.

Kamarajan, C., Porjesz, B., Rangaswamy, M., Tang, Y. Q., Chorlian, D. B., Padmanabhapillai, A., Saunders, R., Pandey, A. K., Roopesh, B. N., Manz, N., Stimus, A. T., and Begleiter, H. (2009). Brain signatures of monetary loss and gain: Outcome-related potentials in a single outcome gambling task. Behav. Brain Res. 197, 62-76.

Knoblich, G., and Sebanz, N. (2008). Evolving intentions for social interaction: from entrainment to joint action. Philos. Trans. R. Soc. Lond. B Biol. Sci. 363, 2021-2031.

Kobayashi, C., Glover, G. H., and Temple, E. (2006). Cultural and linguistic influence on neural bases of 'Theory of Mind': an fMRI study with Japanese bilinguals. Brain Lang. 98, 210-220.

Kobayashi, C., Glover, G. H., and Temple, E. (2007a). Cultural and linguistic effects on neural bases of "theory of dren. Brain Res. 1164, 95-107. from concepts to brain imaging. mind" in American and Japanese chil-

\section{ACKNOWLEDGMENTS}

This work was supported by the National Natural Science Foundation of China [grant number 30870780], Program for New Century Excellent Talents in University and Open Research Fund of the State Key Laboratory of Cognitive Neuroscience and Learning. We thank Professor Lucy Crouppen for her English editing on this manuscript.

Kobayashi, C., Glover, G. H., and Temple, E. (2007b). Children's and adults' neural bases of verbal and nonverbal "theory of mind." Neuropsychologia 45, 1522-1532.

Krämer, U. M., Mohammadi, B. Donamaor, N., Samii, A., and Münte, T. F. (2010). Emotional and cognitive aspects of empathy and their relation to social cognition-an fMRI study. Brain Res. 1311, 110-120.

Leng, Y., and Zhou, X. L. (2010). Modulation of the brain activity in outcome evaluation by interpersonal relationship: an ERP study. Neuropsychologia 48, 448-455.

Leslie, A. M. (1999). "Theory of mind as a mechanism of selective attention," in The New Cognitive Neurosciences, 2nd Edn, ed. M. Gazzaniga (Cambridge: MIT Press), 1235-1247.

Liu, D., Meltzoff, A. N., and Wellman, H. M. (2009a). Neural correlates of beliefand desire-reasoning. Child Dev. 80 1163-1171.

Liu, D., Sabbagh, M. A., Gehring, K. W., and Wellman, H. M. (2009b). Neural correlates of children's theory of mind development. Child Dev. 80, 318-326.

Liu, D., Sabbag, M. A., Gehring, W. J., and Wellman, H. M. (2004). Decoupling beliefs from reality in the brain: an ERP study of theory of mind. Neuroreport 15, 991-995.

Maarten, A. S., Boksem, M. A., and Cremer, D. D. (2010). Fairness concerns predict medial frontal negativity amplitude in ultimatum bargaining. Soc. Neurosci. 5, 118-128.

Mitchell, J. P. (2006). Mentalizing and Marr: an information processing approach to the study of social cognition. Brain Res. 1079, 66-75.

Mitchell, J. P. (2008). Activity in right temporo-parietal junction is not selective for theory-of-mind. Cereb. Cortex 18, 262-271.

Mitchell, J. P. (2009). Inferences about mental states. Philos. Trans. R. Soc. Lond. B Biol. Sci. 364, 1309-1316.

Mobbs, D., Yu, R. J., Meyer, M. Passamonti, L., Seymour, B., Calder, A. J., Schweizer, S., Frith, C. D., and Dalgleishi, T. (2009). A key role for similarity in vicarious reward. Science 324, 900.

Perner, J., Aichhorn, M., Kronbichler, M. Staffen, W., and Ladurner, G. (2006). Thinking of mental and other rep- resentations: the roles of left and right temporo-parietal junction. Soc. Neurosci. 1, 245-258.

Polezzi, D., Daum, I., Rubaltelli, E., Lotto, L., Civai, C., Sartori, G., and Rumiati, R. (2008). Mentalizing in economic decision-making. Behav. Brain Res. 190, 218-223.

Raposo, A., Vicens, L., Clithero, J. A., Dobbins, I. G., and Huettel, S. A. (2010). Contributions of frontopolar cortex to judgments about self, others and relations. Soc. Cogn. Affect. Neurosci. 5, 1-10.

Redcay, E., Dodell-Feder, D., Pearrow, M. J., Mavros, P. L., Kleiner, M., Gabrieli, J. D., and Saxe, R. (2010). Live face-to-face interaction during fMRI: a new tool for social cognitive neuroscience. Neuroimage 50, 1639-1647.

Rilling, J. K., Sanfey, A. G., Aronson, J. A., Nystrom, L. E., and Cohen J. D. (2004). The neural correlates of theory of mind within interpersonal interactions. NeuroImage 22, 1694-1703

Sabbagh, M., and Taylor, M. (2000). Neural correlates of theory-of-mind reasoning: an event-related potential study. Psychol. Sci. 11, 46-50.

Sabbagh, M. A., Moulson, M. C., and Harkness, K. L. (2004). Neural correlates of mental state decoding in human adults: an event-related potential study. J. Cogn. Neurosci. 16, 415-426.

Saxe, R., and Kanwisher, N. (2003). People thinking about thinking people: the role of the temporo-parietal junction in "theory of mind." Neuroimage 19, 1835-1842.

Saxe, R., and Powell, L. J. (2006). It's the thought that counts: specific brain regions for one component of theory of mind. Psychol. Sci. 17, 692-699.

Saxe, R., and Wexler, A. (2005). Making sense of another mind: the role of the right temporo-parietal junction. Neuropsychologia 43, 1391-1399.

Sebanz, N., Bekkering, H., and Knoblich, G. (2006). Joint action: bodies and minds moving together. Trends Cogn. Sci. (Regul. Ed.) 10, 70-76.

Sommer, M., Katrin Döhnel, Sodia, B. Jörg Meinhardt, Thoermer, C., and Hajaka, G. (2007). Neural correlates of true and false belief reasoning. Neuroimage 35, 1378-1384. 
Spezi, M. L., Huang, P. Y., Castelli, F., and Adolphs, R. (2007). Amygdala damage impairs eye contact during conversations withrealpeople.J.Neurosci.27,3994-3997.

Tager-Flusberg, H., and Sullivan, K. (2000).A componential view of theory of mind: evidence from Williams syndrome. Cognition 76, 59-90.

Walter, H., Adenzato, M., Ciaramidaro, A., Enrici, I., Pia, L., and Bara, B. G. (2004). Understanding intentions in social interaction: the role of the anterior paracingulate cortex. J. Cogn. Neurosci. 16, 1854-1863.
Wang, Y.W., Lin, C. D., Yuan, B., Huang, L., Zhang, W. X., and Shen, D. L. (2010). Person perception precedes theory of mind: an event related potential analysis. Neuroscience 170, 238-246

Wang, Y. W., Liu, Y., Gao, Y. X., Chen, J., Zhang, W. X., and Lin, C. D. (2008). False belief reasoning in the brain: an ERP study. Sci. China C Life Sci. 51, 72-79.

Wimmer, H. P., and Perner, J. (1983). Beliefs about beliefs: representation and constraining function of wrong beliefs in young children's under- standing of deception. Cognition 13, 103-128.

Yu, Y., Read, S. J., and Miller, L. C. (2006). A taxonomy of situations from Chinese idioms. J. Res. Pers. 40, 750-778.

Conflict of Interest Statement: The authors declare that the research was conducted in the absence of any commercial or financial relationships that could be construed as a potential conflict of interest.

Received: 26 March 2011; accepted: 08 July 2011; published online: 27 July 2011.
Citation: Wang YW, Zheng YW, Lin CD, Wu J and Shen DL (2011) Electrophysiological correlates of reading the single- and interactive-mind. Front. Hum. Neurosci. 5:64. doi: 10.3389/ fnhum.2011.00064

Copyright (c) 2011 Wang, Zheng, Lin, Wu and Shen. This is an open-access article subject to a non-exclusive license between the authors and Frontiers Media SA, which permits use, distribution and reproduction in other forums, provided the original authors and source are credited and other Frontiers conditions are complied with. 